\title{
Downlink Resource Auction in a Tree Topology Structured Wireless Mesh Network
}

\author{
Zhen Kong, Cheng-Zhong Xu \\ Dept. of Electrical and Computer Engineering \\ Wayne State University \\ Detroit, MI 48202,USA \\ Email: \{zkong,czxu\}@wayne.edu
}

\author{
Yu-Kwong Kwok \\ Dept. of Electrical and Electronic Engineering \\ The University of Hong Kong \\ Hong Kong, China \\ Email: ykwok@hku.hk
}

\begin{abstract}
We analyze the problem of downlink resource allocation in a non-cooperative multi-level tree topology structured wireless mesh network in which a selfish mesh router (MR) may refuse to relay other MRs' traffic so as to improve its own performance at the cost of overall system performance. Based on game theory, we propose an auction framework, where the parent MR serves as the auctioneer while its children MRs act as bidders and compete for time-slots. We derive a payment function from radio resource used for relaying traffic instead of money, so as to simplify the implementation and avoid the possible security problems from monetary payment. We prove the existence and uniqueness of Nash Equilibrium and propose a stochastic best response updating algorithm to allow the bids to iteratively converge to $\mathrm{NE}$ in a practical distributed fashion. Simulation results show the proposed auction algorithm greatly outperforms traditional algorithms in non-cooperative environments.
\end{abstract}

Keywords-Wireless mesh networks, resource allocation, auction algorithms, game theory.

\section{INTRODUCTION}

With the capability of providing universal Internet access services at affordable monetary and infrastructure costs, infrastructure wireless mesh network (WMN) has become one of the key topologies for future wireless networking [1], [18]. In a typical WMN, mesh routers (MRs) form the backbone of the network to provide wireless access for mesh clients (MCs). A special MR, i.e., the gateway (GW), is installed between the mesh backbone and the Internet to provide broadband out-bound access. In WMNs, wireless resource is usually scheduled in a centralized manner [4], that is, the GW serves as a cluster head and determines to resource allocated to each MRs. This centralized approach tend to organize all MRs in a tree structure rooted at the GW. Consequently, to provide broadband Internet services to a remote area, the MRs close to the GW shall work as relay nodes transmitting traffic between the Internet and the MRs far away from the GW.

Among contemporary wireless technologies, IEEE 802.16 standard has been regarded as a promising solution for WMNs due to its capacity of combating channel fading and supporting high data rate. The research and deployment of IEEE 802.16-based WMNs have gained enormous popularity recently [14]. However, one of the key enabling features behind such an infrastructure WMN is that a MR should not only serve the MCs in its own local cell, but also relay traffic for outer level MRs. Unfortunately, this kind of cooperation among MRs is not always practical in reality, especially when these MRs are owned by different profit-maximizing entities. In such a non-cooperative environment, a MR will rationally exhibit selfish behaviors driven by self-interests in that the MR may report a bogus channel information or valuation so as to improve its own benefit [11], or refuse to relay other MRs' traffic because relaying traffic will inevitably consume its own resource and consequently degrade its own performance. This selfish problem is further complicated in a multi-level tree topology WMN environment, where the centralized scheduler at the GW can only impose limited loose control over the distributed remote MRs. Though these non-cooperative behaviors could improve the performance for the selfish MR itself, they may lead to inefficient or unfair resource utilization from the system perspective.

Several recent work has addressed the problem of resource allocation in WMNs. In [4], the authors studied the opportunistic scheduling in an IEEE 802.16j based WiMAX relay networks so as to serve the mobiles in a fair manner and improve the throughput and increase range of WMN effectively. But they didn't take the selfish behavior of mesh routers into account. In [5], the problem of joint routing and scheduling in 802.16 based mesh networks was investigated and an ILP optimization model was proposed to determine a minimum schedule period and maximize the spectrum spatial reuse. While in [3], a joint power-frequency-time resource allocation algorithm was designed to optimize the system performance of a clustered WMN. But these two studies were both based on global optimization, and the competition among different MRs was not considered. In [14], a radio resource management framework for scheduling and admission control in an IEEE 802.16-based WMN was proposed to maximize transmission rate as well as satisfy QoS requirements under power constraint in a wireless MR. It just provided a study of the performance of an individual router; overall system performance was not analyzed. In [17], the throughput maximization problems in a WMN for both cooperative and non-cooperative scenarios were analyzed, and a linear pricing scheme was proposed to combat 
the selfish behavior of non-cooperative MRs. However, the strategy to relay traffic was not considered. In [7], two auction-based approaches were proposed to help relay nodes to determine how to allocate transmission power among users as well as improve fairness and efficiency performances. In their model a relay node itself does not have local users to serve, which is different with our model where a relay MR should serve both local and relay connections.

In this paper, we design a novel auction-based resource allocation scheme. In this scheme, the whole WMN is divided into multiple auction sub-markets. Each sub-market comprises of a parent $\mathrm{MR}$ and its children MRs. The parent MR serves as the auctioneer, who has traffic for its children and grandchildren MRs, while the children MRs act as bidders and compete with each other for the timeslots from their parent. Within this model, we propose a relay auction (RA) algorithm based on a concave utility function, where a MR's bid is a scalar directly associated with its reported valuation, and the payment is proportional to this bid. Though in some auction algorithms [7], [19], the payments are expressed in terms of money, they cannot be easily implemented in practice and may suffer from security problems. In this paper, one of our major contributions is that we associate the payment with the time-slots used by a MR to relay others' traffic. Specifically, to win timeslots allocated by the auctioneer, a bidder must "pay" some wireless resources, i.e., time-slots, and use them to forward its children's traffic. We prove the existence and uniqueness of Nash equilibrium (NE) [15] for the bidding profile. We also present a stochastic bid update algorithm to achieve $\mathrm{NE}$ in a practical situation and prove its convergence. The simulation show that the proposed RA algorithm greatly outperforms traditional algorithms in non-cooperative environments, while having the capability of balancing the efficiency and fairness by adjusting the payment function parameter.

The rest of this paper is organized as follows. We present the system model and auction framework in Section II. In Section III, our novel relay auction approach is proposed. Section IV gives the simulation results. We conclude in Section V.

\section{SySTEM MODEL}

In this paper, we consider an IEEE 802.16-based WMN with tree topology and focus on downlink resource allocation. Such a hierarchical network structure is highly practical and can be used to model a WMN in a rural area [4]. We assume the tree structure has been already determined using link metrics such as Expected Transmission Time (ETT) [16]. The proposed WMN model is represented by a tree graph $G=(V, E)$. Here, $V$ is a set of MRs in the WMN, and $E$ is a set of edges between every parent and child $\mathrm{MR}$, i.e., the wireless links on the routing tree. There are $|V|=N+1 \mathrm{MRs}$ in the tree, where $N$ is the number of
MRs other than the GW. The tree's maximal level is $L$, and the root GW is at level 0 . Specifically, a MR $i \in V$ is at level $l$ if it is $l$ hops away from the GW, and the edge $e(i)$ between node $i$ and its parent MR $P_{i}$ is also at level $l$.

We assume every cell centered on a MR operates under IEEE 802.16 OFDM/TDMA-TDD mode, and each adjacent cell uses a different frequency band. Except the MRs at the farmost level $L$, every MR has both local MC connections served in its local cell and relay connections for its children MRs. The MAC frame is composed of downlink and uplink subframes. Each downlink subframe is composed of $T_{d}$ timeslots, each of which is used for transmission of packets corresponding to one connection. For uplink and downlink transmission using OFDM, each MR uses $M$ subchannels with total bandwidth of $B \mathrm{MHz}$. Without loss of generality, we show in Fig. 1 a part of tree graph which consists of the MR $i$ at level $l$, its parent $P_{i}$ at level $l-1$, its siblings $j \in S_{i}$ with the same parent, and its children MRs $C_{i}$ at level $l+1$. $S_{i}$ is the set of $i$ 's siblings, $N_{i}$ is the number of MRs in $S_{i}$. For the sake of ease, we also let $i \in S_{i}$. For MR $i, a_{i}^{L}$ and $a_{i}^{R}$ are the average number of local packets and relay packets that can be successfully transmitted to its local cell and its children MRs per time-slot, respectively. They can be measured according to channel and traffic conditions.

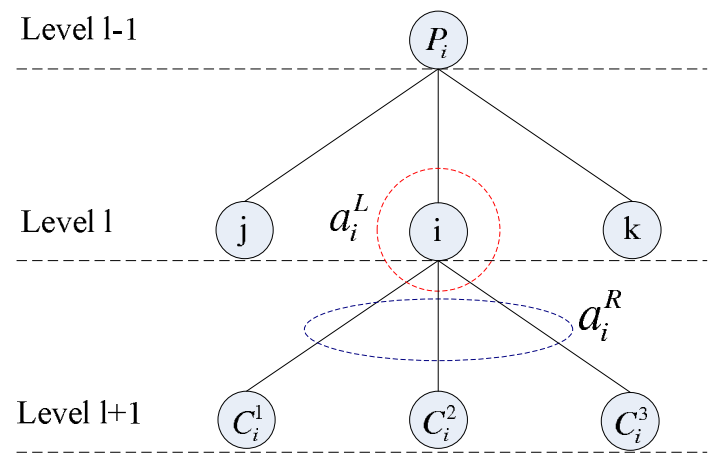

Figure 1: Tree model.

To implement auction, we first divide the WMN into several sub-markets. A sub-market is made up of a MR, its siblings and their parent. For example, in Fig. 1, the MR $i$ 's siblings $S_{i}$ and their parent $P_{i}$ form a sub-market $\Psi_{i}$, where each MR $i$ acts as bidder and competes for time-slots from the auctioneer $P_{i}$ through auction. Each sub-market can operate independently and locally. To be noticed, because the outmost MRs at level $Y$ do not have relay connections, they are not organized into auction sub-markets and we assume they get resources through their parents' scheduling.

In our model, we assume if receiving $t_{i}$ time-slots per frame, MR $i$ 's valuation is $V_{i}^{L}\left(a_{i}^{L}, t_{i}\right)$, thus its valuation function can be fully characterized by the scalar parameter $a_{i}^{L}$. Further, as in [9], we consider to use the following logarithmic function to express MR $i$ 's valuation on its 
received resource, i.e.,

$$
V_{i}^{L}\left(a_{i}^{L}, t\right)=N_{i}^{L} \cdot \log \left(\frac{a_{i}^{L}}{N_{i}^{L}} \cdot t+1\right),
$$

where $N_{i}^{L}$ is the number of MCs served by MR $i$. Thus, $V_{i}^{L}\left(a_{i}^{L}, t\right)$ also represents MR $i$ 's local valuation when it serves $N_{i}^{L}$ local MCs. Similarly, we let $V_{i}^{R}\left(a_{i}^{R}, t\right)$ be MR $i$ 's relay valuation, i.e,

$$
V_{i}^{R}\left(a_{i}^{R}, t\right)=N_{i}^{R} \cdot \log \left(\frac{a_{i}^{R}}{N_{i}^{R}} \cdot t+1\right),
$$

where $N_{i}^{R}$ is the number of its relay connections. We assume that $P_{i}$ has already obtained $a_{i}^{R}$ during the process of routing tree construction. It can also know $N_{i}^{R}$ and $N_{i}^{L}$ according to the buffered traffic.

In an auction, a succinct and expressive bidding language related to bidders' valuation is necessary. According to (1), a bidders valuation function on the allocated resource is actually fully characterized by a single scalar parameter $a_{i}^{L}$. Because $a_{i}^{L}$ is only related to MR $i$ 's local downlink transmission and is not used for WMN tree construction, the auctioneer doesn't know it before auction. Thus, this scalar $a_{i}^{L}$ can be utilized as bidding language and submitted to the auctioneer to represent bidder's valuation, then it leads to an efficient and practical bidding process.

Specifically, during the auction, every bidder $i$ calculates the valuation parameter $a_{i}^{L}$ and submits a bid $b_{i}=\mu_{i}\left(a_{i}^{L_{1}}\right)$ to the auctioneer according to a rationally selfish strategy. After receiving the announced bidding profile $B=$ $\left\{b_{1}, b_{2}, \ldots, b_{N_{i}}\right\}$ from its children, the parent $P_{i}$ knows each bidder's reported valuation, and then calculates the allocation $T=T(B)=\left\{T_{1}, T_{2}, \ldots, T_{i}, \ldots, T_{N_{i}}\right\}$, which represents the number of time-slots allocated to each bidder, as well as the payment $T^{R}=T^{R}(B)=\left\{T_{1}^{R}, T_{2}^{R}, \ldots, T_{i}^{R}, \ldots, T_{N_{i}}^{R}\right\}$ representing the number of time-slots that should be used by MR $i$ to relay its descendant MRs' traffic. Subsequently, the allocation and payment results are transmitted to each bidder. Then, each MR $i$ gets to know $T_{i}^{L}=T_{i}-T_{i}^{R}$ and payment $T_{i}^{R}$.

In a typical auction, the utility of a bidder is the value received by this bidder minus the payment assigned by the auctioneer. However, in our model, to provide practical meanings for payment, we associate the payment with the practical radio resource, i.e., time-slots. Thus, when MR $i$ receives $T_{i}$ time-slots and accept $T_{i}^{R}$ time-slots as payment, its utility for serving local connections is its true valuation when getting $T_{i}^{L}=T_{i}-T_{i}^{R}$ time-slots, i.e.,

$$
\begin{aligned}
& U_{i}^{L}\left(a_{i}^{L}, a_{i}^{R}, T_{i}, T_{i}^{R}\right) \\
= & V_{i}^{L}\left(a_{i}^{L}, T_{i}-T_{i}^{R}\right)-c_{i} \cdot\left(a_{i}^{L} \cdot\left(T_{i}-T_{i}^{R}\right)+a_{i}^{R} \cdot T_{i}^{R}\right),
\end{aligned}
$$

where the second item of (3) is the cost of packet transmission, such as power consumption and so on. $c_{i}$ is the average cost per packet for MR $i$. On the other hand, its relay connections can get a net benefit of

$$
\begin{gathered}
U_{i}^{R}\left(a_{i}^{R}, T_{i}^{R}\right)=V_{i}^{R}\left(a_{i}^{R}, T_{i}^{R}\right) . \\
\text { III. RELAY AUCTION }
\end{gathered}
$$

One well known auction is the Vickrey-Clarke-Groves (VCG) auction, which has been regarded as one of the most effective mechanisms to induce truth-revealing strategies [12]. However, the VCG auction does not consider fairness performance. Furthermore, it just applies to the problem where bidders have a quasilinear utility function [2]. From (3), we see the utility function cannot be expressed as the difference between the valuation and payment, implying that they are not quasi-linear. Thus, the VCG method cannot be applied here. We need to propose another new auction approach.

Generally, the auctioneer wants to maximize the aggregate utility of it transmitted packets. At the same time, it also need to enforce the bidder to relay other's traffic through payments. Specifically, the more resource a bidder requests, the higher payment it should pay to the auctioneer. Thus, we define the payment as a function increasing with the bidding scalar $b_{i}$, and propose a relay auction (RA) algorithm in submarket, which is formalized as follows.

\section{A. Auctioning Mechanism}

1) Bidding rule: In a sub-market $\Psi_{i}$, bidder $i$ submits a scalar value $b_{i}$ to its parent $P_{i}$, and let $\mathbf{b}=\left\{b_{i}\right.$ : $\left.b_{\min } \leq b_{i} \leq b_{\max }\right\}$.

2) Payment rule: If bidder $i$ gets $T_{i}$ time-slots, it must use $T_{i}^{R}=f\left(b_{i}\right) \cdot T_{i}$ time-slots for relaying its children's traffic, where $f\left(b_{i}\right)$ is increasing with $b_{i}$, and $0 \leq f\left(b_{i}\right) \leq 1$. Here, we consider a linear payment function: $f\left(b_{i}\right)=\frac{b_{i}}{A}$, where $A$ is a payment parameter and $A>b_{\max }$.

This payment scheme has two implications. First, it can restrict bidder's selfish behavior due to the fact that when given a payment parameter $A$, larger resource request means more obtained time slots should be used for relay. Thus, a rational bidder must request resource strategically. On the other hand, for a fixed bid, a larger $A$ implies that larger portion of resource can be used for local traffic; while more resource are utilized for relay connection in terms of a smaller $A$. Therefore, it can adjust the tradeoff between efficiency and fairness in the system, which is further verified through simulations in Section IV.

3) Allocation rule: We assume the auctioneer's objective is to maximize the aggregate utilities of all local connections and relay connections in its sub-market, 


$$
\begin{aligned}
& \text { i.e., } \\
& T_{*}=\left\{T_{1}^{*}, T_{2}^{*}, \cdots, T_{N}^{*}\right\} \\
& =\arg \max _{\left\{T_{i}\right\}} \sum_{i \in S_{i}} U_{i}^{L}\left(T_{i}-T_{i}^{R}\right)+U_{i}^{R}\left(T_{i}^{R}\right) \\
& =\arg \max _{\left\{T_{i}\right\}} \sum_{i \in S_{i}}\left(N_{i}^{L} \cdot \log \left(\frac{b_{i}}{N_{i}^{L}} \cdot\left(T_{i}-T_{i}^{R}\right)+1\right)\right. \\
& +N_{i}^{R} \cdot \log \left(\frac{a_{i}^{R}}{N_{i}^{R}} \cdot T_{i}^{R}+1\right) \\
& \left.-c_{i} \cdot\left(b_{i} \cdot\left(T_{i}-T_{i}^{R}\right)+a_{i}^{R} \cdot T_{i}^{R}\right)\right)
\end{aligned}
$$

subject to

$$
\begin{aligned}
& \text { (I) } \sum_{i}\left(T_{i}^{L}+T_{i}^{R}\right)=T_{d} \\
& \text { (II) } T_{i}^{R}=f\left(b_{i}\right) \cdot T_{i} .
\end{aligned}
$$

where the constraint (I) implies the time-slots constraint in one frame, and the constraint (II) is the payment function.

\section{B. Nash Equilibrium}

When the bidder $i$ and its siblings compete with each other in a sub-market $\Psi_{i}$, our proposed relay auction can be formulated as a strategic non-cooperative game with $N_{i}$ players, i.e., $\Gamma \triangleq\left[S_{i},\left\{b_{i}\right\}_{i \in S_{i}},\left\{U_{i}^{L}(\mathbf{B})\right\}_{i \in S_{i}}\right]$, where $S_{i}$ is the player set, $\left\{b_{1}, \cdots, b_{N_{i}}\right\}$ is the action profile, and $U_{i}^{L}$ is the utility function of player $i$.

A useful solution concept of such a game is called a Nash equilibrium (NE) [15], which is a bidding profile $B^{*}$ where no MR wants to deviate unilaterally, i.e.,

$$
U_{i}^{L}\left(b_{i}^{*} ; \mathbf{b}_{-i}^{*}\right) \geq U_{i}^{L}\left(b_{i} ; \mathbf{b}_{-i}^{*}\right), \quad \forall i \in S_{i}, \quad \forall b_{i} \geq 0,
$$

where $\mathbf{b}_{-i}$ is the bidding profile of bidder $i$ 's opponents. We first establish the existence of the NE.

Theorem 1. In $\Psi_{i}$, there exists a bidding profile $B^{*}=$ $\left\{b_{1}^{*}, \cdots, b_{N_{i}}^{*}\right\}$ to achieve Nash equilibrium in the relay auction.

Proof: A Nash equilibrium $B^{*}$ exists in the game if the following two conditions are satisfied for all $i \in S_{i}$ :

1) the bidding strategy $b_{i}$ is a nonempty, convex and compact subset of some Euclidean space $\Re^{N}$; and

2) $U_{i}^{L}(\mathbf{B})$ is continuous in $\mathbf{B}$ and quasi-concave in $b_{i}$

The first condition is easily satisfied because we have $b_{i} \in$ $\left[b_{\min }, b_{\max }\right]$. For utility function $U_{i}^{L}$, because $\frac{\partial^{2} U_{i}^{L}}{\partial b_{i}^{2}}<0$, it is strictly concave on $b_{i}$. As a concave function is also quasi-concave, the second condition is then satisfied. Thus, the existence of NE is proven.

We then construct MR $i$ 's best response function (for fixed $\left.\mathbf{b}_{-i}\right)$ as:

$$
\beta_{i}\left(\mathbf{b}_{-i}\right)=\left\{b_{i} \mid b_{i}=\arg \max _{\widetilde{b_{i}} \geq 0, i \in S_{i}} U_{i}^{L}\left(\widetilde{b_{i}} ; \mathbf{b}_{-i}\right)\right\} .
$$

Then, similar to the proof in [6], we show the uniqueness of NE in the following.
Theorem 2. In $\Psi_{i}$, there exists a unique $N E B^{*}=$ $\left\{b_{1}^{*}, \cdots, b_{N_{i}}^{*}\right\}$ in the relay auction.

Proof: We assume that NE is not unique, and there exists at least another one $\tilde{B}=\left\{\tilde{b}_{1}, \cdots, \tilde{b}_{N_{1}}\right\}$, and $B^{*} \neq \tilde{B}$. According to the definitions of $\mathrm{NE}$ and the best response function, if given $b_{-i}, b_{i}^{*}$ and $\tilde{b}_{i}$ must satisfy:

$$
\begin{aligned}
b_{i}^{*} & =\max _{b_{i}^{*}} U_{i}^{L}\left(b_{i}^{*}, b_{-i}\right), \\
\tilde{b}_{i} & =\max _{\tilde{b_{i}}} U_{i}^{L}\left(\tilde{b}_{i}, b_{-i}\right) .
\end{aligned}
$$

However, because $U_{i}^{L}$ is concave and differentiable, for a fixed $b_{-i}$, there is a unique best strategy $b_{i}$ that satisfies $b_{i}=\max _{b_{i}} U_{i}^{L}\left(b_{i}, b_{-i}\right)$. These contradict the assumption that $B^{*} \neq B$. Thus, $B^{*}=\tilde{B}$, and there is a unique NE in this auction sub-market.

\section{Iterative Best Response Update Algorithm to Achieve NE}

In our model, it is difficult to get the close-form $\beta_{i}\left(\mathbf{b}_{-i}\right)$ and $U_{i}^{L}\left(b_{i}, \mathbf{b}_{-i}\right)$. However, because the nonlinear constrained optimization problem defined by (5) can be efficiently solved by numerical methods, and there exists a unique NE in the auction, and MR $i$ 's utility function $U_{i}^{L}$ in (3) is differentiable and concave related to $b_{i}$, we can still design an stochastic bid updating algorithm to achieve NE based on each bidder's local information and the limited feedback received from the auctioneer. Although it is still hard to obtain the exact expression of $U_{i}^{L}\left(b_{i}, \mathbf{b}_{-i}\right)$, we can regard the optimization problem to find the best response in (7) as a stochastic approximation problem [13], and then get recursive solutions for the best response through the stochastic gradient form of Robbin-Monro algorithm [13] and the finite difference (FD) method. The key idea is to let the auctioneer gives the bidders some additional information besides the auction results so that the bidder can use it to estimate the gradient of its utility by FD method and then update its bid according to Robbin-Monro algorithm.

Specifically, at time $t$, when the auctioneer $P_{i}$ receives $\mathbf{b}$ and calculates the allocation $T(\mathbf{b})$ and payment $T^{R}(\mathbf{b})$, it also calculates $T_{i}^{L+}=T_{i}^{L}\left(\mathbf{b}+c_{t} \cdot \mathbf{e}_{i}\right)$ and $T_{i}^{L-}=T_{i}^{L}(\mathbf{b}-$ $\left.c_{t} \cdot \mathbf{e}_{i}\right)$. Here, $c_{t} \rightarrow 0$ is a FD interval and $\mathbf{e}_{i}$ be the standard unit vector in the $i^{\text {th }}$ coordinate direction. Then they are all sent to bidder $i$. With this knowledge, MR $i$ can estimate its utility gradient $\nabla_{b_{i}(t)} U_{i}\left(b_{i}(t), b_{-i}(t)\right)$ by FD method as follows:

$$
\begin{aligned}
\nabla_{b_{i}(t)} U_{i}(\mathbf{b}) & =\frac{U_{i}^{L}\left(\mathbf{b}+c_{t} \cdot \mathbf{e}_{i}\right)-U_{i}^{L}\left(\mathbf{b}-c_{t} \cdot \mathbf{e}_{i}\right)}{2 c_{t}} \\
& =\frac{U_{i}^{L}\left(T_{i}^{L+}\right)-U_{i}^{L}\left(T_{i}^{L-}\right)}{2 c_{t}} .
\end{aligned}
$$

It further apply Robbin-Monro algorithm to update the best response by

$$
b_{i}(t+1)=b_{i}(t)+a_{t} \cdot \nabla_{b_{i}(t)} U_{i}(\mathbf{b}),
$$


where the gain sequence $a_{t}>0, a_{t} \rightarrow 0, \Sigma_{t=o}^{\infty}\left(a_{t}\right)=\infty$. Then bidder $i$ submits bid $b_{i}(t+1)$ again at time $t+1$. We let the bids update asynchronously, e.g., the auctioneer $P_{i}$ only calculates bidder $i$ 's additional information at time $\left\{i, i+N_{i}, i+2 N_{i}, \cdots\right\}$. Then the NE can be achieved gradually. The stochastic best response update algorithms for the auctioneer and the bidders are formalized as follows in Algorithm 1, and Algorithm 2, respectively.
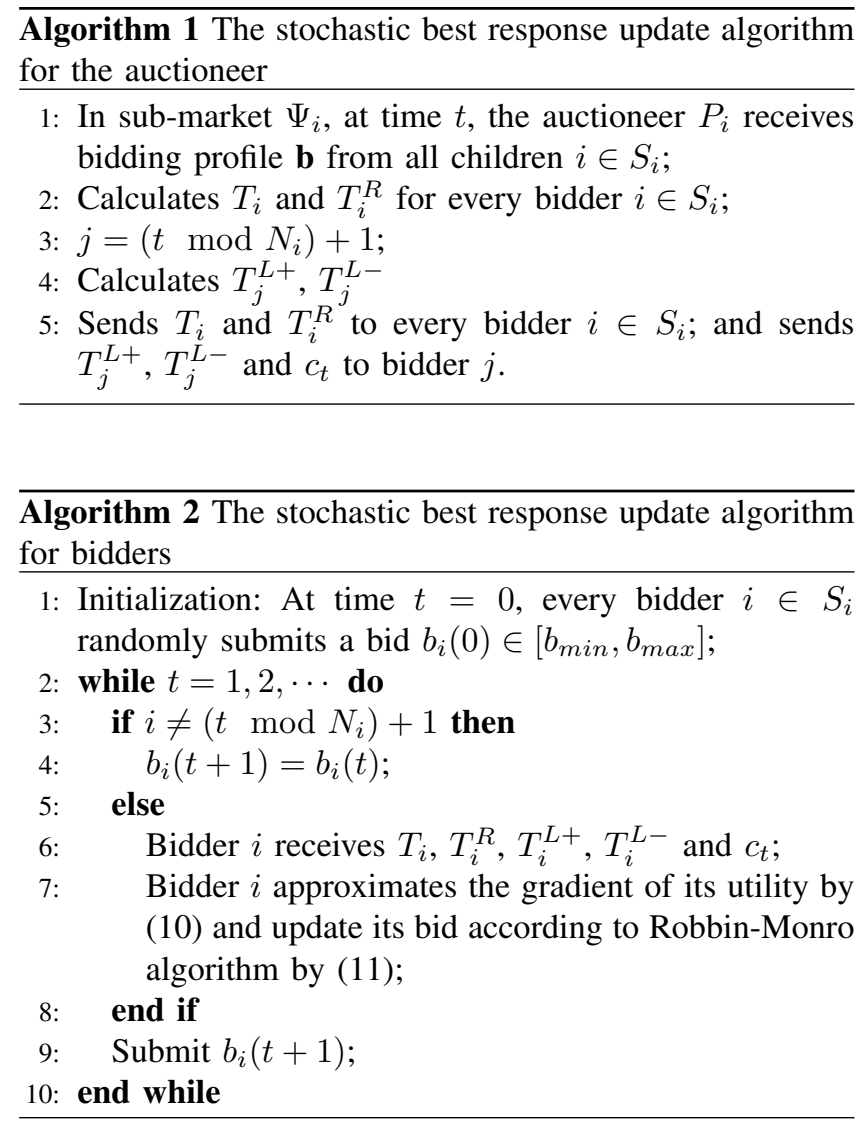

Theorem 3. For each bidder $i \in \Psi_{i}$, under any initial condition $\boldsymbol{b}(0)$, the sequence $\{\boldsymbol{b}(t)\}$ converges weakly (i.e., in probability) to $N E B^{*}$.

Proof: We first interpolate the iterates $\mathbf{b}(t)$ into a continuous time interpolation process $\mathbf{b}^{\epsilon_{t}}(t)=\left\{b_{i}^{\epsilon_{t}}(t)\right\}$ with interpolation intervals $\epsilon_{t}$, and define $b_{i}^{\epsilon}(t)$ as follows:

$$
\bar{b}_{i}^{\epsilon_{t}}(t)= \begin{cases}b_{i}(0) & t<0 \\ b_{i}(n) & n \epsilon_{t}-\epsilon_{i} \leq t \leq n \epsilon_{i}, n=1,2, \cdots .\end{cases}
$$

Thus, as shown in [13], when $\epsilon_{t} \rightarrow 0, b_{i}^{\epsilon_{t}}(t)$ converges in trajectory to $\bar{b}_{i}(t)$. Here, $\bar{b}_{i}(t)$ is the solution of the ordinary differential equation (ODE) function

$$
\frac{d \bar{b}_{i}(t)}{d t}=\nabla_{\bar{b}_{i}(t)} U_{i}\left(\bar{b}_{i}(t), b_{-i}(t)\right)
$$

Because $U_{i}\left(b_{i}(t), b_{-i}(t)\right)$ is bounded and quasi-concave with respect to $b_{i}$, the ODE in (13) is asymptotically Liapunov stable. Consequently, as $\epsilon_{t} \rightarrow 0$, the sequence $b_{i}(t)$ converges weakly to an optimizer of its utility function, i.e., Nash equilibrium $b_{i}^{*}$.

The convergence of the stochastic bid update algorithm is also verified from Fig. 2, where $a_{1}^{L}=10, a_{2}^{L}=9$, and $A=90$. We can see the bids converge to NE eventually.

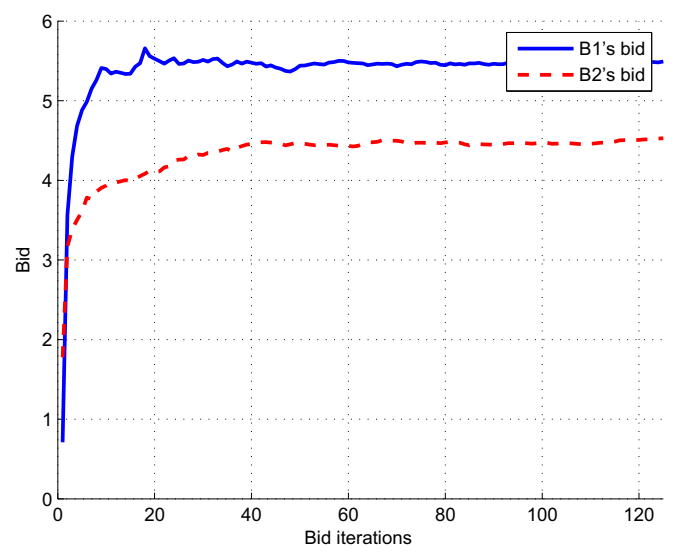

Figure 2: Convergence of bids to NE.

\section{Performance Results}

In this section, we evaluate the performance of the auction algorithm through simulations. In the simulated tree topolgy WMN, we assume each MR has 64 subcarriers with a total frequency band of $10 \mathrm{MHz}$. The frequency bands for adjacent MRs are non-coverlapping and frequency reuse is utilized. The DL-subframe length is $2 \mathrm{~ms}$. The wireless channel is modeled as six-path frequency-selective Rayleigh slow fading channel; each path is simulated by Clark's fading model and suffers from different Rayleigh fading with the maximum Doppler frequency of $30 \mathrm{~Hz}$. Because the coherence time $33.3 \mathrm{~ms}$ is much larger than the DLsubframe length, the channel condition can be regarded as static during the iterative bidding period to achieve NE. This is a realistic assumption in a WMN because the MRs are immobile. Furthermore, we assume the average SNR is identical for all MCs in a cell. Like [4], we do not model packet arrival process at the GW and MRs, and assume the GW has infinite backlogged traffic for each MC in WMN and the downlink mesh tree is constructed by using routing algorithms, such as ETT by [16]. We assume each parent MR has at most 3 children, and there are $10 \mathrm{MCs}$ in each cell. Then the traffics generated at GW can be transmitted across the WMN and reach MCs through other MR's relay. $b_{\text {min }}$ and $b_{\max }$ are 1 and 20, respectively, which also represent the minimum and the maximum successfully transmittable packets per time slots. 
We first compare our proposed RA algorithm with two traditional cooperative resource allocation methods, i.e., proportional fairness (PF) [10], and a global optimization method (GLB) which maximizes the aggregate utilities of all MRs based on centralized scheduling, in non-cooperative environments. In this setting, the maximal tree level $L$ is from 2 to 5 , and the payment parameter $A$ is 90 . Notice that in a cooperative situation which is unrealistic in the operating environment considered in this paper, all MRs report their true valuations to their parents and relay other's traffic according to scheduling results. However, in a noncooperative environment, a selfish MR may report a bogus valuation parameter to increase its own local benefit without regard to the overall system performance [11] and relay less traffic to others. In our simulations, we assume in each sub-market, there is a MR who always reports a higher valuation in the case of using PF and GLB algorithms. From Fig. 3, we first notice that the average throughput per MR decreases with the size of WMN, due to the downlink transmission capacity limit of the GW. Another reason is that more packets may be dropped due to increased wireless transmission error along the longer path when the network expands. Furthermore, the average throughput per MR of PF and GLB are both much lower than that of RA algorithm due to the fact that less traffics are relayed to remote MRs. While in RA algorithm, a selfish MR may not benefit from bidding higher value because this will also incur higher payment. Consequently, the system performance is maintained in $\mathrm{NE}$ state during auctions.

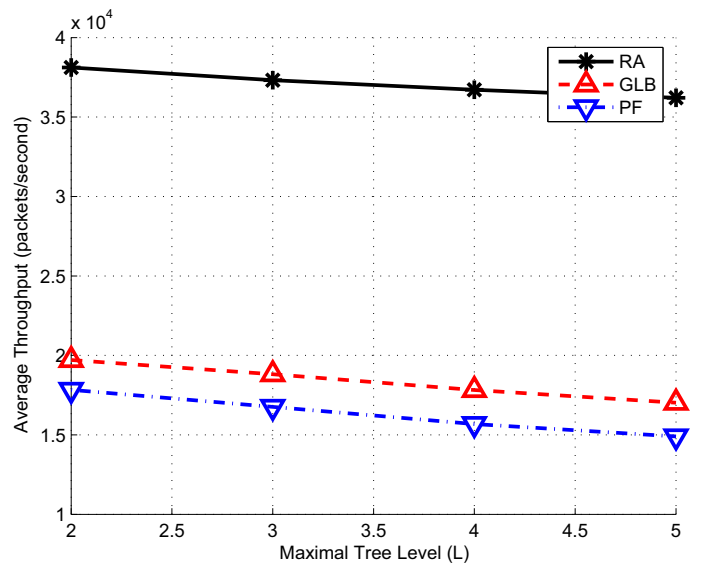

Figure 3: Average throughput per MR with the change of network size in non-cooperative situations.

We then illustrate the throughput and fairness performances of RA in terms of payment parameter, where the maximal tree level $L$ is 2, and compare them with the performances of GLB and PF in cooperative situations. In Fig. 4, we notice that the average throughput per MR of RA algorithm increases with the payment parameter $A$.
Although GLB and PF generate higher throughput than RA algorithm, the throughput of RA achieves almost $95 \%$ of that for GLB with the increase of payment parameter, e.g., $A>100$, even in non-cooperative environments. This is because the time-slots used for relay will decrease if the payment parameter becomes larger, then more resources are utilized to server local MCs' traffic. Because local MCs are usually closer to the MR and generally experience better transmission conditions than relay connections, the overall system throughput increases when more resources are used for local traffic. We then investigate the fairness performance in terms of Jain fairness index [8]. We can see in Fig. 5 that the Jain fairness index for RA algorithm is dependent on the payment parameter $A$. RA achieves its highest index value 0.735 when $A=122$, which is about $95 \%$ of PF's fairness index value. Thus, the proposed RA algorithm can be used to strike a proper balance between efficiency and fairness by adjusting payment function parameter. Furthermore, when utilizing RA algorithm in non-cooperative environments, the efficiency and fairness performances are comparable with those obtained by GLB and PF in operative situations.

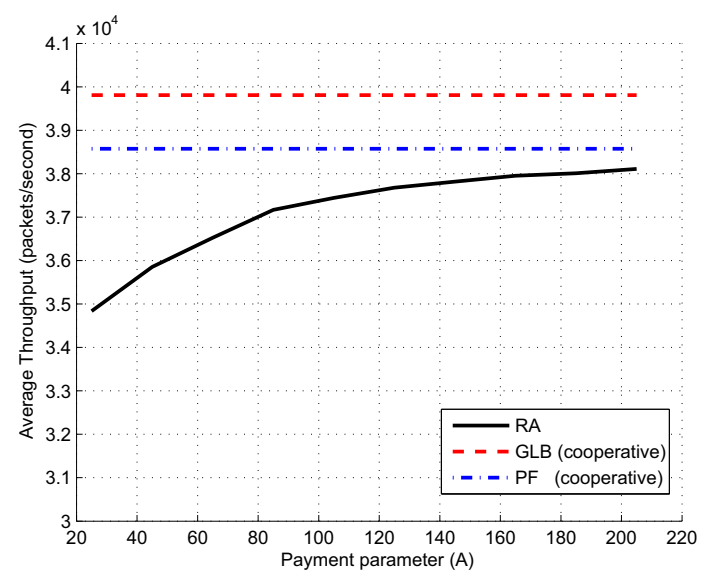

Figure 4: Average throughput per MR with the change of payment parameter.

\section{Conclusions}

In this paper, we study the downlink resource allocation problem in a non-cooperative IEEE 802.16 tree topology based wireless mesh network. To motivate selfish MRs to relay other's traffic, we divide the entire network into multiple auction sub-markets and design an auction framework to let rationally selfish MRs compete with their siblings for time-slots from their parent. Based on it, we further propose a novel relay auction algorithm, where the payment is designed as the time-slots used for relaying traffic instead of money, and the allocation policy is to maximize both local and relay connections' utilities in the sub-market. After proving the existence and uniqueness of NE, we also 


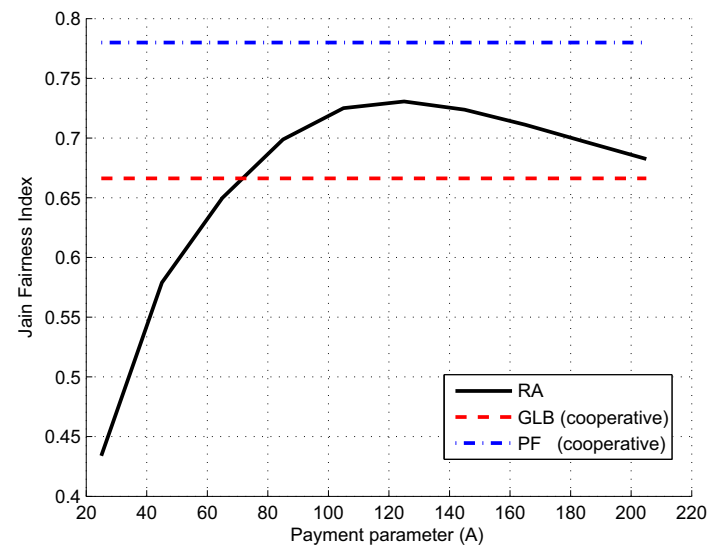

Figure 5: Jain fairness index with the change of payment parameter.

propose a stochastic best response update algorithm to allow bidders to iteratively update their bids based on their local information and the limited feedback from the auctioneer and prove its convergence to NE. We then compare the performances of the RA algorithm with traditional GLB and PF algorithms through simulations, which indicate that the RA algorithm excels GLB and PF in non-cooperative situations. It also can achieve competitive performance in terms of resource allocation efficiency with high payment parameter $A$, while demonstrating good fairness performance with small payment parameter, so as to strike a proper balance between efficiency and fairness in the network.

\section{Acknowledgements}

This work was supported in part by U. S. NSF under grants CRI-0808232, CNS-0702488, CNS-0914330, and CCF-1016966.

\section{REFERENCES}

[1] I. F. Akyildiz and X. Wang, "A Survey on Wireless Mesh Networks," IEEE Communications Mag., vol. 43, no. 9, pp. S23-S30, Sept. 2005.

[2] L. M. Ausubel and P. Milgrom, "The Lovely but Lonely Vickrey Auction," in Combinatorial Auctions, P. Cramton, Y. Shoham, and R. Steinberg (eds.), Chapter 1, MIT Press, Cambridge, MA, 2006.

[3] H. T. Cheng and W. Zhuang, "Joint Power-Frequency-Time Resource Allocation in Clustered Wireless Mesh Networks," IEEE Network, pp. 45-51, Jan/Feb. 2008.

[4] S. Deb, V. Mhatre and V. Ramaiyan, "WiMAX Relay Networks: Opportunistic Scheduling to Exploit Multiuser Diversity and Frequency Selectivity," in Proc. of 14th ACM International Conference on Mobile Computing and Networking (MobiCom 2008), San Francisco, CA, USA, Sept. 2008.
[5] J. El-Najjar, C. Assi, and B. Jaumard, "Joint Routing and Scheduling in WiMAX-Based Mesh Networks," IEEE Trans. Wireless Communications, vol.9, no.7, pp.2371-2381, July 2010.

[6] G. He, S. Gault, M. Debbah, and E. Altman,"Distributed Power Allocation Game for Uplink OFDM Systems," in Proc. of 6th Intl. Symposium on Modeling and Optimization in Mobile, Ad Hoc, and Wireless Networks (WiOPT 2008), pp. 515-521, Berlin, Germany, Mar.-Apr. 2008.

[7] J. Huang, et al., "Auction-Based Resource Allocation for Cooperative Communications," IEEE J. Select. Areas Communications, vol.26, no. 7, pp.1226-1237, Sept. 2008.

[8] R. Jain, D. Chiu and W. Hawe, "A Quantitative Measure of Fairness and Discrimination for Resource allocation in Shared Computer System,” DEC Technical Report 301, 1984.

[9] Z. Jiang, Y. Ge, and Y. Li, "Max-Utility Wireless Resource Management for Best-Effort Traffic," IEEE Trans. Wireless Communications, vol. 4, no. 1, pp. 100-111, Jan. 2005.

[10] F. Kelly, "Charging and Rate Control for Elastic Traffic," Eur. Trans. Telecommun., vol. 8, no. 1, pp. 33-37, Jan. 1997.

[11] Z. Kong, Y. K. Kwok, and J. Wang, "On the Impact of Selfish Behaviors in Wireless Packet Scheduling," in Proc. of the IEEE International Conference on Communications 2008 (ICC 2008), pp. 3253-3257, Beijing, China, May 2008.

[12] V. Krishna, Auction Theory, Academic Press, London, UK, 2002.

[13] H. J. Kushner and G. G. Yin, Stochastic Approximation and Recursive Algorithms and Applications, Springer, 2003.

[14] D. Niyato, E. Hossain, and V. K. Bhargava, "Scheduling and Admission Control in Power-Constrained OFDM Wireless Mesh Routers: Analysis and Optimization," IEEE Trans. Wireless Communications, vol.6, no.10, pp. 3738-3748, Oct. 2007.

[15] M. J. Osborne, An Introduction to Game Theory, New York: Oxford University Press, 2004.

[16] J. Padhye, R. Draves and B. Zill,"Routing in multi-radio, multi-hop wireless mesh network," in Proc. of 10th ACM International Conference on Mobile Computing and Networking (Mobicom 2004), Philadelphia, PA, USA, Sept. 2004.

[17] Y. Song, C. Zhang, and Y. Fang, "Joint Channel and Power Allocation in Wireless Mesh Networks: A Game Theoretical Perspective," IEEE J. Select. Areas Communications, vol. 26, no. 7, pp. 1149-1159, Sept. 2008.

[18] P. Zhou, X. Wang and R. Rao, "Asymptotic Capacity of Infrastructure Wireless Mesh Networks." IEEE Trans. Mobile Computing, vol. 7, no. 8, pp. 1011-1024, Aug. 2008.

[19] X. Zhou, et al., "eBay in the Sky: Strategy-Proof Wireless Spectrum Auctions," in Proc. of 14th ACM International Conference on Mobile Computing and Networking (MobiCom 2008), San Francisco, CA, USA, Sept. 2008. 